\title{
CARACTERÍSTICAS DE INGRESO DE PACIENTES CON ASMA EN UN PROGRAMA DE REHABILITACIÓN PULMONAR
}

\section{INCOME GHARACTERISTICS OF PATIENTS WITH ASTHMA IN A PULMONARY REHABILITATION PROGRAM}

\author{
Stefanie Tonguino-Rosero ${ }^{1}$ \\ Liésery Arroyave-Lozano ${ }^{2}$ \\ Xiomara Muñoz-Obando ${ }^{3}$ \\ Estefania Espitia-Sandoval ${ }^{4}$ \\ Jessica Vásquez-Hurtado ${ }^{5}$ \\ Julian Rivera-Motta ${ }^{6}$ \\ Jhonatan Betancourt-Peña ${ }^{7}$
}

Fecha de Recepción: 06 de junio de 2016

Fecha de Aceptación: 18 de diciembre de 2016

\section{Citar como:}

Tonguino-Rosero S, et al. (2016). Características de ingreso de pacientes con asma en un programa de Rehabilitación pulmonar. Revista Movimiento Científico 10(2): 21-29.

Tonguino-Rosero S, et al. (2016). Características de ingreso de pacientes con asma en un programa de Rehabilitación pulmonar. Revista Movimiento Científico [en línea] 2016, [fecha de consulta: dd//mm/aaaa]; 10(2): 21-29. Disponible desde: http://revistas. iberoamericana.edu.co/index.php/Rmcientifico/issue/archive.

1 Fisioterapeuta. Especialista en Fisioterapia Cardiopulmonar. Docente Facultad de Salud y Rehabilitación. Institución Universitaria Escuela Nacional del Deporte (Cali, Colombia). Docente Facultad de Salud, Escuela de Rehabilitación Humana. Universidad del Valle (Cali, Colombia).

2 Fisioterapeuta. Institución Universitaria Escuela Nacional del Deporte (Cali, Colombia).

Fisioterapeuta. Institución Universitaria Escuela Nacional del Deporte (Cali, Colombia).

Fisioterapeuta. Institución Universitaria Escuela Nacional del Deporte (Cali, Colombia).

Fisioterapeuta. Institución Universitaria Escuela Nacional del Deporte (Cali, Colombia).

6 Fisioterapeuta. Especialista en Rehabilitación Cardíaca y Pulmonar. Magíster en Ciencias Básicas. Docente Facultad de Salud y Rehabilitación. Institución Universitaria Escuela Nacional del Deporte (Cali, Colombia). Docente Facultad de Salud, Universidad Santiago de Cali (Cali, Colombia). Clínica Neumológica del Pacífico.

7 Fisioterapeuta. Especialista en Rehabilitación Cardíaca y pulmonar, Magíster en Administración, Docente Facultad de Salud y Rehabilitación Institución Universitaria Escuela Nacional del Deporte (Cali, Colombia). Docente Facultad de Salud, Escuela de Rehabilitación Humana. Universidad del Valle (Cali, Colombia). Cardioprevent SAS. Correo electrónico: johnnatanbp@hotmail.com 


\section{RESUMEN}

Introducción: el asma es una enfermedad pulmonar generalmente caracterizada por la inflamación crónica de las vías respiratorias. Objetivo: describir las características de ingreso y valoración de los pacientes con asma de un Programa de Rehabilitación Pulmonar en Cali. Materiales y métodos: estudio descriptivo de tipo transversal. Se recolectaron

datos de los pacientes mediante una evaluación de la historia clínica sobre las características sociodemográficas, clínicas, ansiedad y depresión; se realizó el test de caminata de los 6 minutos. Resultados: de 11 pacientes incluidos en el estudio, 9 eran mujeres con edad promedio de 61.8 años. La comorbilidad más frecuente fue la hipertensión arterial seguida del hipotiroidismo. Dentro de los fármacos más utilizados se encuentran los B2 acción corta en un $91 \%$. Se evidenció patrón obstructivo en la espirometría post broncodilatador. La media de saturación de oxígeno antes de realizar el test de caminata de 6 minutos fue de 96\%, al final del Test fue de 90,2\%; La media en la distancia recorrida al iniciar el programa de rehabilitación pulmonar es de 293,3m $\pm 110,2 \mathrm{~m}$. Conclusiones: La causa principal de visitas de los pacientes asmáticos a urgencias es la falta de control de los síntomas, pruebas como el test de caminata de los 6 minutos permiten establecer la limitación de la funcionalidad de los pacientes con asma.

Palabras clave: Asma, Rehabilitación, Disnea.

\section{ABSTRACT}

Background: Asthma is a lung disease usually characterized by chronic inflammation of the airways. Objective: Describe the characteristics of admission, diagnosis and treatment of patients with Asthma, who get in a Pulmonary Rehabilitation program in Cali city. Materials and methods: It's a descriptive and cross-sectional study, patient data were collected by analysing medical records on sociodemographic, clinical, anxiety and depression and six minutes walk test. Results: Of 11 patients included in the study, 9 were women with an average age of 61.8 years. The most frequent comorbidity is high blood pressure, with a ratio 4.5 out of 10 , followed by hypothyroidism. Among the most commonly used drugs are short-acting B2 with 91\%. The average oxygen saturation before making the walk test of six minutes was $96 \%$ at the end of the test was $90.2 \%$. The average distance travelled by patients with asthma when starting a pulmonary rehabilitation program is $\pm 293,3 \mathrm{~m} 110,2 \mathrm{~m}$. Conclusions: The main cause of visits to the emergency room asthma patients is the lack of control of symptoms, Tests such as 6 minutes walk test establish limitation of the functionality of patients with asthma.

Keywords: Asthma, Rehabilitation, Dyspnoea.

\section{INTRODUCGIÓN}

El asma es una enfermedad pulmonar generalmente caracterizada por la inflamación crónica de las vías respiratorias. Se diagnostica por la historia de síntomas respiratorios como sibilancias, dificultad para respirar, opresión en el pecho y tos, que varían en el tiempo y en intensidad, junto con la limitación del flujo aéreo espiratorio variables. Estos episodios se asocian generalmente a la obstrucción generalizada pero variable en el flujo aéreo pulmonar que es frecuentemente reversible espontáneamente o con el tratamiento. Los factores que influyen en el riesgo de desarrollar asma pueden ser relacionados a factores primariamente genéticos y los otros son usualmente factores ambientales (Reddel, Barnes, Bateman, Becker $\&$ Bel, 2012).

En la actualidad, aproximadamente 300 millones de personas en el mundo padecen asma, produciéndose alrededor de 180.000 muertes anuales (Álvarez, Adames, Rodríguez, Barrios \& Wever, 2010). Los estudios epidemiológicos en América Latina han revelado prevalencias variables de 5,7 a $16,5 \%$ que 
pueden deberse a las distintas metodologías empleadas en los estudios o a la influencia de diversos factores de riesgo (Bacab \& Alpuche, 1997).

Para confirmar el diagnóstico de asma, se realizan pruebas diagnósticas que permiten cuantificar la gravedad de la enfermedad, monitorizar la evolución y objetivar la respuesta al tratamiento. La espirometría es la técnica más utilizada por su sencillez y coste; se conoce como la "prueba reina" en el estudio del asma. Con ella medimos los volúmenes y flujos pulmonares generados en una maniobra de espiración máxima voluntaria, que puede ser simple o forzada. La capacidad vital forzada (FVC), es el máximo aire que puede ser espirado de forma forzada tras una inspiración máxima. El volumen espiratorio máximo en el primer segundo (FEV1), es el volumen espirado en el primer segundo desde que comienza la espiración. Está disminuido en los procesos obstructivos como es el caso del asma. El cociente FEV1/FVC, es el porcentaje de la capacidad vital forzada que se espira en el primer segundo. En condiciones normales este porcentaje es del $75-80 \%$ y se encuentra disminuido en los procesos obstructivos. En general, se consideran valores normales para el FEV 1 cifras entre el $80 \%$ y el $120 \%$. El patrón obstructivo se considera leve cuando el valor porcentual del FEV1 está entre 65-80\%, moderado entre el 50-64\%, o grave si es menor del 50\%). (Sánchez, Rocamora, Asensi, Jiménez, Iborra, Benítez, 2004).

La conducta adoptada por la mayoría de los países para el tratamiento del asma bronquial a partir del año 1990 se basa en el uso de broncodilatadores a demanda, como el salbutamol inhalado, en pacientes con síntomas ligeros y poco frecuentes; sin embargo la disnea es un síntoma frecuente que requiere mayor control y las recomendaciones (Moore, Bleecker, Curran-Everett, Erzurum, Ameredes, Bacharier \& Dweik, 2007) sugieren ejercicio y soporte educacional al paciente y familiares para optimizar la función pulmonar y disminuir los síntomas y exacerbaciones. No obstante en nuestro medio es poco frecuente la remisión de estos pacientes a programas de rehabilitación pulmonar y se conoce poco sobre las condiciones de ingreso a dichos programas.
Este estudio tiene como objetivo describir las características de ingreso y valoración de los pacientes con asma de un Programa de Rehabilitación Pulmonar en Cali, Colombia.

\section{MATERIALES Y MÉTODOS}

Estudio descriptivo de tipo transversal realizado en una población de pacientes diagnosticados con asma, quienes ingresaron al programa de Rehabilitación Pulmonar en una clínica en la ciudad de Cali (Colombia) durante el periodo comprendido de julio de 2012 y junio de 2014. Teniendo en cuenta la poca remisión de pacientes diagnosticados por especialistas en neumología se vincularon por conveniencia pacientes diagnosticados con asma que ingresaban por primera vez al programa de rehabilitación pulmonar, con información de pruebas de función pulmonar en el último mes y que firmaran el consentimiento informado.

Los criterios de exclusión fueron: pacientes con complicaciones de comorbilidades en el último mes o durante la rehabilitación, que requirieron manejo por urgencias u hospitalización, pacientes con déficit cognitivo, problemas en locomoción o cualquier condición que comprometiera realizar test de caminata de los 6 minutos.

Este estudio fue aprobado por el Comité de Ética Humana de la Institución Universitaria Escuela Nacional del Deporte (acta IV. 4,1-01-03), garantizando la confidencialidad, autonomía y todas las medidas de seguridad necesarias para la aplicación de los instrumentos de evaluación.

El diseño metodológico comprendió una revisión teórica de guías que soportaran la intervención de pacientes con asma en programas de rehabilitación pulmonar (Gómez \& Durán, 2012; Bingisser et al., 2001; Palange et al, 2007).

La aplicación de los instrumentos de medición fue realizada por un fisioterapeuta especialista en rehabilitación cardiaca y pulmonar antes del ingreso de los pacientes al programa de rehabilitación pulmonar. 
A través de un cuestionario diseñado por los investigadores se indagó acerca de características sociodemográficas, clínicas y de tratamiento farmacológico.

Se registraron las variables: edad, género, estado civil, estrato socioeconómico, hábito de fumar, número paquetes/año, exposición al humo de leña, exposición ocupacional, número de hospitalizaciones en el último año, índice de masa corporal (IMC); también se determinó la necesidad de oxígeno domiciliario según indicación del médico que remitía al programa (Manresa, Sena, \& Caballol, 2001; Betancourt-peña \& Tonguino-Rosero, 2016), patologías asociadas, exámenes diagnósticos, tratamiento farmacológico, pulso-oximetría en reposo.

La severidad de la disnea se estableció por medio de la escala de 5 grados de la Medical Research Council (MRC) (Fletcher, 1960). Se registraron los valores de la CVF, VEF1, VEF1/CVF en porcentaje del predicho obtenidos en la espirometría diagnóstica post broncodilatador y resultados de la encuesta HADS de ansiedad y depresión. Posteriormente se les realizó dos test de caminata de los 6 minutos y se eligió le mejor a todos los pacientes para establecer la distancia recorrida según las guías de manejo de la American Thoracic Society (2002). Este procedimiento tuvo una duración estimada de 1 hora 30 minutos.

\section{Análisis estadístico:}

Las variables fueron registradas en una base de datos diseñada en Excel 2010 y se sistematizó en el paquete estadístico EPIINFO 2000.

Se hizo un análisis descriptivo univariado para cada uno de los datos; las variables cualitativas se presentan en frecuencia y porcentaje; asumiendo la normalidad de las variables con la prueba Shapiro-Wilk las variables cuantitativas se presentan en medias \pm desviación estándar.

\section{RESULTADOS}

Entre Julio de 2012 a Junio de 2014 ingresaron 14 pacientes al programa de rehabilitación pulmonar con diagnóstico de asma; 11 pacientes cumplían los criterios de inclusión y otros 3 pacientes fueron excluidos por presentar otras comorbilidades respiratorias como EPOC.

Las características sociodemográficas y clínicas al ingreso de los pacientes se encuentran en la tabla 1. La edad media de los pacientes fue de $61,8 \pm 20$ años, siendo el $81,8 \%$ del género femenino. En el estado civil 5 pacientes $(45,4 \%)$ eran casados, seguido de soltero, unión libre y viudo con $2(18,8 \%)$ para cada uno de los casos.

En cuanto al estrato socioeconómico el $54,5 \%$ de los pacientes pertenece al estrato 3, 27,3\% al estrato 2, el 9,1\% al estrato 1 y 4 para cada uno. En relación al hábito de fumar el $45.5 \%$ nunca había fumado, 54,5\% son exfumadores y un paciente $(9,1 \%)$ continuaba fumando al momento del ingreso. La mediana de paquetes año de cigarrillo fue de 13,8 con una amplitud intercuartílica de 45,8. La exposición al humo de leña estuvo presente en 2 pacientes (18,2\%). Se observó que 9 pacientes $(81,8 \%)$ presentaron visitas a urgencias en el último año por complicaciones respiratorias, de los cuales 6 pacientes $(54,5 \%)$ fueron hospitalizados un promedio de 6 días $\pm 4,1$.

Respecto a la patología asociada más frecuente se encontró la hipertensión arterial en 5 pacientes $(45,5 \%)$.

Las características de las pruebas de función pulmonar y capacidad funcional se muestran en la tabla 2. Para la espirometría en los pacientes con asma se presentaron patrones obstructivos en todos los pacientes. La distancia recorrida en el TC6M al iniciar el programa de rehabilitación pulmonar fue de 293,3 con una desviación estándar de 110,2 metros, para la distancia predicha en el TC6M la media fue de 490,2 con una desviación estándar de 124. 
Tabla 1. Características sociodemográficas y clínicas

\begin{tabular}{|c|c|c|}
\hline Variable & Pacientes $=11$ & $\begin{array}{c}\text { Porcentaje } \\
(\%)\end{array}$ \\
\hline \multicolumn{3}{|l|}{ Género } \\
\hline Masculino & 2 & 18,2 \\
\hline Femenino & 9 & 81,8 \\
\hline \multicolumn{3}{|l|}{ EPS } \\
\hline Subsidiado & 1 & 9,1 \\
\hline Contributivo & 10 & 90,9 \\
\hline \multicolumn{3}{|l|}{ Estrato } \\
\hline 1 & 1 & 9,1 \\
\hline 2 & 3 & 27,3 \\
\hline 3 & 6 & 54,5 \\
\hline 4 & 1 & 9,1 \\
\hline \multicolumn{3}{|l|}{ Fumó en el último año } \\
\hline $\mathrm{Si}$ & 6 & 54,5 \\
\hline No & 5 & 45,5 \\
\hline Peso (kg) & 11 & $64,5 \pm 11 *$ \\
\hline Talla (metros) & 11 & $1,5 \pm 0,1^{*}$ \\
\hline \multicolumn{3}{|l|}{ IMC } \\
\hline Delgadez & 1 & 9,1 \\
\hline Normal & 3 & 27,3 \\
\hline Sobrepeso & 4 & 36,4 \\
\hline Obesidad & 3 & 27,3 \\
\hline \multicolumn{3}{|l|}{ Uso de oxígeno domiciliario } \\
\hline $\mathrm{Si}$ & 9 & 69,2 \\
\hline No & 4 & 30,8 \\
\hline \multicolumn{3}{|l|}{ Comorbilidades } \\
\hline Hipertensión arterial & 5 & 45,5 \\
\hline Diabetes & 1 & 9,1 \\
\hline Hipotiroidismo & 1 & 9,1 \\
\hline Fibrilación ventricular & 1 & 9,1 \\
\hline
\end{tabular}

* Valores presentados en Media y desviación estándar.

Fuente: elaboración propia (2016)

Los exámenes diagnósticos previos al ingreso evidenciaron que el $82 \%$ de los pacientes tienen espirometría pre y post broncodilatador, $46 \%$ test de caminata de los 6 minutos, $36,4 \%$ rayos x de tórax, 27,3\% difusión de monóxido de carbono, 18,3\% TAC de tórax y polisomnografía cada una, 9,1\% para espirometría simple, volúmenes pulmonares, ecocardiograma, prueba de ejercicio cardiopulmonar integrada y gases arteriales.
Tabla 2. Características de la función pulmonar y capacidad funcional

\begin{tabular}{lcc}
\hline \multicolumn{1}{c}{ Variables } & Casos & $\begin{array}{c}\text { Media } \pm \\
\text { Desviación estándar }\end{array}$ \\
\hline Espirometría & & \\
VEF1 \% & & \\
CVF \% & 11 & $43,3 \pm 18$ \\
VEF1/CVF \% & 11 & $66,8 \pm 28,1$ \\
Disnea reposo Borg & 11 & $65,0 \pm 4,5$ \\
fatiga en miembros inferiores & 11 & $0,27 \pm 0,9$ \\
MRC al inicio del programa & 11 & $0,45 \pm 1,2$ \\
VO2 estimado al final del & 11 & $2,8 \pm 1,1$ \\
TC6M & 11 & $8,4 \pm 1,8$ \\
MET TC6M & 11 & $2,4 \pm 0,5$ \\
Distancia recorrida en el & 11 & $293,3 \pm 110,2$ \\
TC6M & 11 & $490,2 \pm 124$ \\
Distancia predicha del TC6M & 11 & $95,5 \pm 3$ \\
SaO2 reposo & 11 & $90,2 \pm 7,6$ \\
SaO2 Final TC6M & 11 & $5,3 \pm 6,6$ \\
Porcentaje de desaturación & 11 & $1,1 \pm 3,8$ \\
Ansiedad & 11 & $6,7 \pm 3,6$ \\
Depresión & & \\
\hline
\end{tabular}

Fuente: elaboración propia (2016)

Las características del tratamiento farmacológico se describen en la tabla 3. Los fármacos más usados por los pacientes con asma fueron los $\beta 2$ de acción corta y anticolinérgicos en presentación inhalador de dosis medida (IDM), con un $46 \%$ para cada uno de los fármacos.

Tabla 3. Características del tratamiento farmacológico

\begin{tabular}{lcc}
\hline \multicolumn{1}{c}{ Fármacos } & Casos & $\%$ \\
\hline B2 acción corta & 5 & $46 \%$ \\
Anticolinérgico & 5 & $46 \%$ \\
B2 acción prolongada/antiinflamatorio & 4 & $36,4 \%$ \\
Antiinflamatorio inhalado & 3 & $27,3 \%$ \\
Oxigenoterapia & 3 & $27,3 \%$ \\
Calcio antagonista & 2 & $18,2 \%$ \\
Antileucotrieno & 2 & $18,2 \%$ \\
Betabloqueador & 2 & $18,2 \%$ \\
Otros & 1 & $9,1 \%$ \\
\hline
\end{tabular}

Fuente: elaboración propia (2016) 


\section{DISCUSIÓN}

En nuestro contexto, este estudio en es pionero en describir las características basales de un grupo de pacientes con diagnóstico de asma que ingresaron a un programa de rehabilitación pulmonar. La escases de estudios previos, probablemente, obedece a que la mayoría de programas de rehabilitación pulmonar se dirigen a pacientes con EPOC, así como a la carencia de registro y análisis de información por parte de los fisioterapeutas y demás profesionales que participan en dichos programas (Gómez V \& Duran D. 2010).

Para este estudio se encontró que la mayoría de los pacientes eran mujeres, casadas, de estrato socioeconómico 3 y con una edad media de 62 años, esta condición se explica porque la prevalencia de asma en la edad adulta se observa con mayor frecuencia en los pacientes de 40 años o más, siendo significativamente mayor en el género femenino (Moral y Cols. 2003).

Llama la atención que al menos la mitad de los pacientes $(54,5 \%)$ reportaron haber tenido hospitalizaciones en el último año por exacerbaciones de la enfermedad, esa misma cantidad reportaron haber fumado durante el último año. Estos resultados fueron similares a los de Cisneros y cols (2006) quienes encontraron que el 36\% de los pacientes asmáticos que consultaban a neumología habían tenido ingresos hospitalarios, además el $82 \%$ requirieron de visitas a urgencias, siendo la falta de control de la enfermedad el principal motivo de este tipo de consultas.

Lo anterior permite reconocer que los pacientes asmáticos deben mejorar el control de la enfermedad para prevenir exacerbaciones, las cuales en algunos casos podrían llevarlos a la muerte. De esta manera, los programas de rehabilitación pulmonar se convierten en una estrategia clave en el tratamiento de este tipo de pacientes ya que incluyen un manejo integral que involucra procesos educativos donde el paciente participa como agente activo.

Se encontró que más de la mitad de la población estudiada (54.5\%) fumó en el último año, es de resaltar ese alto porcentaje de pacientes asmáticos fumadores, ya que actualmente se sabe que el humo del cigarrillo es un factor de riesgo de exacerbaciones del asma. Además, existen muchas campañas antitabaco entre la población general, de igual manera, este estudio arrojó que ningún paciente de los que ingresó al programa de rehabilitación pulmonar realizaba actividad física; Valdivia G, en el 2000, indicó que las fluctuaciones en el nivel de actividad física pueden guardar relación con la creciente tendencia en el asma y enfermedades atópicas, también sugirió que la reducción en la movilización de volúmenes pulmonares asociados a estilos de vida sedentarios, podrían conducir a la modificación de la capacidad elástica de la musculatura lisa bronquial, a un estrechamiento en la vía aérea, a su vez el sedentarismo incrementaría también el tiempo de exposición a aero-alérgenos en el hogar y podrían facilitar el contacto con otros factores involucrados en la respuesta inmune.

Regalado-Pineda y cols. 2005 en ciudad de México estudiaron a 919 sujetos con asma entre 14 y 86 años, reportando un VEF1 menor en los pacientes que tenían antecedentes de tabaco; es de resaltar que en nuestro estudio el $54.5 \%$ de los pacientes fumaban hasta por lo menos un año antes de ingresar a rehabilitación pulmonar, esto posiblemente pueda influir en el compromiso del VEF1 de los pacientes; sin embargo, es una situación que amerita un análisis más profundo en futuras investigaciones.

Por otro lado, los medicamentos más usados fueron los broncodilatadores de acción corta seguido de los anticolinérgicos. Oller-Legra y cols 2011, estudiaron a 196 pacientes con asma bronquial de edades entre 25 a 44 años y encontraron resultados similares donde el $80 \%$ de los pacientes usaban broncodilatador de acción corta y un anticolinérgico.

Asimismo, se pudo observar que la totalidad de los pacientes fueron remitidos por la especialidad de neumología. La remisión por pare de esta especialidad fue similar en estudio realizado en Cuba, donde se estudiaron 20 pacientes con asma bronquial, de ambos sexos y diferentes grupos de edad, diagnosticados como tales, fueron remitidos de las consultas de neumología e inmunoalergia (Meneses T, 1999). 
Durante la duración de este estudio el ingreso de los pacientes fue en promedio 1 paciente con diagnóstico de asma por primera vez a rehabilitación pulmonar cada dos meses, lo cual refleja un número muy bajo de pacientes; sin embargo, durante la revisión de la literatura no se encontraron estudios donde se pueda comparar el promedio de ingreso mensual de pacientes a programas de rehabilitación pulmonar ya sea de asma o de otra patología respiratoria como la EPOC.

En cuanto a la condición clínica, la media de disnea en reposo según Borg fue de 0,27 y la fatiga en miembros inferiores de 0,45 . Lo anterior concuerda con los resultados del estudio de Martínez-Moragón y cols 2008, donde la media de la disnea basal según Borg fue de 0,56; encontrándose entonces que hay una hipopercepción o escasa percepción de la disnea, esto se convierte en una circunstancia que se da aproximadamente en un $13 \%$ de los asmáticos, y tiene una importancia crucial, puesto que se ha visto relacionada con las crisis de asma fatal o casi fatal, y con el asma de difícil control.

La percepción de disnea en el asma es el principal síntoma de estos pacientes y el que más altera su calidad de vida. La cuantificación de la disnea en la escala MRC se relaciona mejor con la calidad de vida que las variables funcionales basales, de hecho su percepción puede ser muy desigual entre pacientes con similar grado de obstrucción. La escala MRC es la recomendada por SEPAR por su sencillez y relación con las tareas diarias, la media de la percepción de disnea en reposo según MRC en este estudio para pacientes asmáticos fue de 2,8 por otro lado, según Gallego y cols, la percepción de disnea del paciente asmático no se correlaciona bien con el grado de obstrucción respiratoria medido en la espirometría.

Solo dos pacientes realizaron la prueba con oxígeno, que eran los que usaban oxigeno domiciliario, al final esos dos pacientes y otro que tuvo una caída en su saturación de más de 4\%; Según la Asociación Americana de Tórax (ATS) la oximetría de pulso debe ser incluida como monitoreo, porque es necesaria para los aspectos de seguridad del examen, para evitar exponer a los pacientes a hipoxemia. Una caída significativa de la saturación es aquella mayor o igual a 4\%, lo anterior es la explicación por la cual solo un paciente requirió oxigeno suplementario.

Algunos autores (Leal F.D.E, Parra M, Segura N.H y cols, 2006) encontraron que la frecuencia de ansiedad de acuerdo con la escala $\mathrm{HAD}$ fue del $30 \%$ en pacientes diagnosticados con asma. La frecuencia de depresión en el grupo 1, de acuerdo con la escala HAD, fue del $12 \%$ de los pacientes diagnosticados; esto contrasta un poco con nuestro estudio ya que los pacientes con asma obtuvieron una media en la evaluación de ansiedad de $1,1 \pm 3.8$ y una media en la evaluación de depresión $6,7 \pm 3,6$; en nuestro estudio se observa que de los 11 pacientes involucrados ninguno presentaba un caso clínico de depresión o ansiedad. Teniendo en cuenta que en la escala HADS se considera normal de 0 a 7 , dudoso de 8 a 10 y problema clínico a partir de 11 para ansiedad y depresión (Galindo O y cols. 2015).

El test de caminata de los 6 minutos es la prueba de capacidad aeróbica y funcional más utilizada en los programas de rehabilitación (Hodgkin, Celli \& Connors, 2000). Para este estudio los pacientes presentaban una distancia media por debajo de valores encontrados en otros estudios (Hallstrand, Bates, \& Schoene, 2000; Lucas, \& Platts-Mills, 2005); sin embargo, esto puede deberse a que esta población presentaban mayor deterioro en su clase funcional y sintomatología respiratoria.

Es válido considerar que una limitante de este estudio fue el mínimo número de pacientes reclutados; esto podría servir como una base para realizar futuras investigaciones en pacientes con asma que asisten a programas de rehabilitación pulmonar.

\section{CONGLUSIONES}

La falta de control de los síntomas es la principal causa de exacerbaciones por parte del $50 \%$ de los pacientes que ingresaron al programa de rehabilitación pulmonar; el tratamiento farmacológico mantiene el control del asma con efectos adversos mínimos en los pacientes; los medicamentos más usados fueron lo B2 de acción corta, seguido por los anticolinérgicos. Las 
pruebas como el test de caminata de los 6 minutos y la disnea MRC permitió establecer la marcada limitación de la funcionalidad de los pacientes y la necesidad de implementar intervenciones orientadas a sus necesidades. La rehabilitación pulmonar es una intervención que permite mejorar la capacidad funcional, la calidad de vida, la fatiga y la disnea de pacientes con asma.

\section{AGRADECIMIENTOS}

A la Dirección Técnica de Investigaciones, al profesor Mg. Hugo Hurtado y Mg. Juan Carlos Ávila por los aportes realizados en el análisis de la información.

\section{REFERENCIAS}

ATS Committee on Proficiency Standards for Clinical Pulmonary Function Laboratories. (2002). ATS statement: guidelines for the six-minute walk test. American Journal of Respiratory and Critical Care Medicine, 166(1), 111.

Álvarez, A. J., Adames, D., Rodríguez, J., Barrios, E. E., \& Wever, R. (2010). Infiltrado celular en pacientes asmáticos y su relación con la severidad del asma. Salus, 14(1), 26-30.

Bacab, M. A. B., \& Alpuche, N. E. A. (1997). Prevalencia de asma en niños escolares de Mérida, Yucatán. Rev Panam Salud Pública, 2(5), 299

Betancourt-Peña, J., \& Tonguino-Rosero, S. (2016). Impacto de la oxigenoterapia domiciliaria en la capacidad funcional de pacientes con enfermedad pulmonar obstructiva crónica. Rehabilitación, 50(1), 13-18.

Bingisser, R. M., Joos, L., Fruhauf, B., Caravatti, M., Knoblauch, A., \& Villiger, P. M. (2001). Pulmonary rehabilitation in outpatients with asthma or chronic obstructive lung disease. Swiss medical weekly, 131(27/28), 407-411.

Cisneros, C., Antón, E., Casanova, A., Cobos, M. R., Moreno, R. G., \& Ancochea, J. Características clínico-funcionales de los pacientes asmáticos en una consulta monográfica de neumología. Respiratoria, 5.

Fletcher, C. (1960). Standardised questionnaire on respiratory symptoms: a statement prepared and approved by the MRC Committee on the Aetiology of Chronic Bronchitis (MRC breathlessness score). BMJ, 2, 1665.

Gallego, M. C., Samaniego, J., Alonso, J., Sánchez, A., Carrizo, S., \& Marín, J. M. (2002). Disnea en la EPOC: relación de la escala MRC con la disnea inducida en las pruebas de marcha y de ejercicio cardiopulmonar máximo. Archivos de Bronconeumología, 38(3), 112-116.
Galindo Vázquez, O., Benjet, C., Juárez García, F., Rojas Castillo, E., Riveros Rosas, A., Aguilar Ponce, J. L., et al. (2015). Propiedades psicométricas de la Escala Hospitalaria de Ansiedad y Depresión (HADS) en una población de pacientes oncológicos mexicanos. Salud mental, 38(4), 253-258.

Gómez, V., \& Durán, D. (2012). Effects of a Pulmonary Rehabilitation Program for Severe Asthma: Case Presentation. Revista Ciencias de la Salud, 10(2), 253-264.

Hallstrand, T. S., Bates, P. W., \& Schoene, R. B. (2000). Aerobic conditioning in mild asthma decreases the hyperpnea of exercise and improves exercise and ventilatory capacity. CHEST Journal, 118(5), 1460-1469.

Hodgkin, J. E., Celli, B. R., \& Connors, G. L. (Eds.). (2000). Pulmonary rehabilitation: guidelines to success. Lippincott Williams \& Wilkins.

Leal, F. D. E., Román, M. P., Méndez, N. H. S., Alberto, D., Nicolás, T., Díaz, D. M., et al. (2006). Ansiedad y depresión en asmáticos adultos en comparación con sujetos sanos. Revista Alergia México, 53(6), 201-206.

Lucas, S. R., \& Platts-Mills, T. A. (2005). Physical activity and exercise in asthma: relevance to etiology and treatment. Journal of Allergy and Clinical Immunology, 115(5), 928-934.

Manresa, J. M., Sena, F., \& Caballol, R. (2001). El control de la oxigenoterapia domiciliaria en un hospital comarcal. Archivos de Bronconeumología, 37(7), 237-240.

Martínez-Moragón, E., Perpiñá, M., Fullana, J., Macián, V., Lloris, A., \& Belloch, A. (2008). Perception of dyspnea and treatment adherence in asthmatic patients. Archivos de Bronconeumología (English Edition), 44(9), 459-463.

Meneses Terry, M. R. (1999). Programa de rehabilitación respiratoria en pacientes asmáticos: repercusión sobre las pruebas funcionales respiratorias. Revista Cubana de Medicina, 38(3), 178-182.

Moore, W. C., Bleecker, E. R., Curran-Everett, D., Erzurum, S. C., Ameredes, B. T., Bacharier, L., et al. (2007). Characterization of the severe asthma phenotype by the national heart, lung, and blood institute's severe asthma research program. Journal of Allergy and Clinical Immunology, 119(2), 405-413.

Moral, V. P., Gutiérrez, F. Á., Clarà, P. C., Barroso, N. C., Viña, A. L., Rosselló, M. L., \& Jiménez, J. Q. (2003). Guía española para el manejo del asma. Arch bronconeumol, 39(Supl 5), 3-42.

Oller-Legrá, E., Acosta-Pérez, Y., \& Baquero-Suárez, J. M. (2011). Tratamiento y seguimiento de adultos con asma bronquial en las clínicas de la capital de Namibia. Medisan, 15(3), 356-362. 
Palange, P., Ward, S. A., Carlsen, K. H., Casaburi, R., Gallagher, C. G., Gosselink, R., et al. (2007). Recommendations on the use of exercise testing in clinical practice. European Respiratory Journal, 29(1), 185-209.

Reddel H, Barnes N, Barnes P, Bateman E, Becker A, Bel E. (2012). Global Strategy for Asthma Management and Prevention, Global Initiative for Asthma (GINA).

Regalado-Pineda, J., Gómez-Gómez, A., Ramírez-Acosta, J., \& Vázquez-García, J. C. (2005). Efecto del tabaquismo, los síntomas respiratorios y el asma sobre la espirometría de adultos de la Ciudad de México. Salud pública de méxico, 47(5), 327-334.

Sánchez, E. S., Rocamora, L. P., Asensi, J. V., Jiménez, J. S., Iborra, M. I., Benítez, M. F., \& Ortega, J. S. (2004). Consenso de asma, neumología y alergia pediátrica (borrador). Allergologia et immunopathologia, 32(3), 104-118.

Valdivia, G. (2000). Asma bronquial y enfermedades atópicas como problema emergente de Salud Pública: nuevas hipótesis etiológicas. La experiencia de sociedades desarrolladas. Revista médica de Chile, 128(3), 339-346. 